Journal for ImmunoTherapy of Cancer

\title{
Propensity score-weighted analysis of chemotherapy after PD-1 inhibitors versus chemotherapy alone in patients with non-small cell lung cancer (WJOG10217L)
}

Ryoji Kato, ${ }^{1}$ Hidetoshi Hayashi (i) , ${ }^{1}$ Yasutaka Chiba, ${ }^{2}$ Eriko Miyawaki, ${ }^{3}$ Junichi Shimizu, ${ }^{4}$ Tomohiro Ozaki, ${ }^{5}$ Daichi Fujimoto, ${ }^{6}$ Ryo Toyozawa, ${ }^{7}$ Atsushi Nakamura, ${ }^{8}$ Toshiyuki Kozuki, ${ }^{9}$ Kentaro Tanaka, ${ }^{10}$ Shunsuke Teraoka, ${ }^{11}$ Kazuhiro Usui, ${ }^{12}$ Kazumi Nishino, ${ }^{13}$ Osamu Hataji, ${ }^{14}$ Keiichi Ota, ${ }^{15}$ Noriyuki Ebi, ${ }^{16}$ Sho Saeki, ${ }^{17}$ Yuki Akazawa, ${ }^{18}$ Motoyasu Okuno, ${ }^{19}$ Nobuyuki Yamamoto, ${ }^{11}$ Kazuhiko Nakagawa ${ }^{1}$

To cite: Kato R, Hayashi $\mathrm{H}$, Chiba Y, et al. Propensity score-weighted analysis of chemotherapy after PD-1 inhibitors versus chemotherapy alone in patients with non-small cell lung cancer (WJOG10217L). Journal for ImmunoTherapy of Cancer 2020;8:e000350. doi:10.1136/jitc-2019-000350

- Additional material is published online only. To view please visit the journal online (http://dx.doi.org/10.1136/jitc2019-000350).

Accepted 20 January 2020

Check for updates

(c) Author(s) (or their employer(s)) 2020. Re-use permitted under CC BY-NC. No commercial re-use. See rights and permissions. Published by BMJ.

For numbered affiliations see end of article.

Correspondence to Dr Hidetoshi Hayashi; hidet31@med.kindai.ac.jp

\begin{abstract}
Background Studies have suggested that chemotherapy after immune checkpoint inhibitors may confer an improved response for non-small cell lung cancer (NSCLC). However, potential selection bias in such studies has not been addressed. We therefore applied propensity score analysis to investigate the efficacy of chemotherapy after PD-1 inhibitor treatment (CAP) compared with chemotherapy alone.
\end{abstract}

Methods We conducted a retrospective observational cohort study for patients treated at 47 institutions across Japan between April 1, 2014 and July 31, 2017. Eligible patients had advanced or recurrent NSCLC who have undergone chemotherapy. Patients subsequently treated with chemotherapy (docetaxel with or without ramucirumab, S-1 or pemetrexed) either after PD-1 inhibitor therapy (CAP cohort) or alone (control cohort) were included. The primary end point was objective response rate (ORR). Inverse probability weighting (IPW) was applied to adjust for potential confounding factors. Results A total of 1439 patients (243 and 1196 in the CAP and control cohorts, respectively) was available for unadjusted analysis. Several baseline characteristicsincluding age, histology, EGFR or ALK genetic alterations, and brain metastasis-differed significantly between the two cohorts. After adjustment for patient characteristics with the IPW method, ORR was $18.9 \%$ for the CAP cohort and $11.0 \%$ for the control cohort (ORR ratio $1.71 ; 95 \% \mathrm{Cl}$ 1.19 to 2.46; $p=0.004)$. IPW-adjusted Kaplan-Meier curves showed that median progression-free survival (PFS) for the CAP and control cohorts was 2.8 and 2.7 months (IPW-adjusted HR 0.95; $95 \% \mathrm{Cl} 0.80$ to $1.12 ; \mathrm{p}=0.55$ ), and median overall survival (OS) was 9.2 and 10.4 months (IPW-adjusted HR 1.05; $95 \% \mathrm{Cl} 0.86$ to $1.28 ; \mathrm{p}=0.63$ ), respectively.

Conclusions After accounting for selection bias by propensity score analysis, CAP showed a significantly higher ORR compared with chemotherapy alone, with the primary end point of ORR being achieved. However, these results did not translate into a PFS or OS advantage, suggesting that prior administration of PD-1 inhibitors may result in a synergistic antitumor effect with subsequent chemotherapy, but that such an effect is transient. CAP therefore does not appear to achieve durable tumor control or confer a lasting survival benefit.

\section{BACKGROUND}

The development of immune checkpoint inhibitors (ICIs) has led to a major shift in the treatment of advanced non-small cell lung cancer (NSCLC). Phase III studies of patients with advanced NSCLC who experienced disease progression during or after platinum-based chemotherapy found that the programmed cell death-1 (PD-1) inhibitors nivolumab and pembrolizumab, as well as the programmed cell death-ligand 1 (PDL1) inhibitor atezolizumab, had durable antitumor activity and significantly prolonged overall survival (OS) compared with docetaxel. ${ }^{1-4}$ However, most patients eventually experience disease progression during ICI treatment.

Randomized controlled trials (RCTs) have demonstrated the efficacy of docetaxel, pemetrexed or docetaxel plus ramucirumab in the second-line setting for patients with advanced NSCLC who have undergone prior chemotherapy. ${ }^{5-8}$ In addition, a phase III trial showed the non-inferiority of S-1 relative to docetaxel for East Asian patients with previously treated advanced NSCLC. ${ }^{9}$ These chemotherapy regimens are therefore frequently administered in routine clinical 
practice for patients who experience disease progression during ICI therapy.

Retrospective studies of patients with NSCLC have recently suggested that the objective response rate (ORR) for salvage chemotherapy after ICIs is higher relative to historical data or to the last chemotherapy regimen administered before ICIs. ${ }^{1011}$ However, despite the highly selected populations of patients who received sequential ICIs and chemotherapy, no study has addressed potential selection bias. Although a prospective RCT would be the gold standard for verification of these findings with minimal systematic bias, such a trial is not feasible because the administration of an ICI alone or in combination with chemotherapy is now widely recognized as a standard first-line treatment for patients with advanced NSCLC. ${ }^{12-14}$

Propensity score analysis was designed to eliminate selection bias due to measured patient characteristics that affect both treatment and outcomes in observational studies. ${ }^{15-17}$ A treatment effect estimated from observational databases can provide complementary evidence to support the results of RCTs, given that patients enrolled in RCTs are often highly selected and at low risk, yielding results not generalizable to all real-world clinical settings. ${ }^{16} 17$

We have therefore now performed a multicenter retrospective observational cohort study to evaluate with the use of propensity score analysis whether chemotherapy after PD-1 inhibitor treatment (CAP) has a greater antitumor effect compared with chemotherapy alone.

\section{METHODS}

\section{Study design and patients}

We performed a search of electronic medical records for patients with advanced or recurrent NSCLC treated at 47 affiliated institutions of West Japan Oncology Group (WJOG). Eligible patients had histologically or cytologically confirmed advanced NSCLC who received cytotoxic chemotherapy as a first-line treatment. Patients with recurrent disease who had received curative surgery or chemoradiotherapy were included. The following regimens were not counted as a line of therapy: preoperative or postoperative adjuvant chemotherapy, chemotherapy associated with curative radiotherapy, epidermal growth factor receptor (EGFR) tyrosine kinase inhibitors (TKIs) for EGFR mutation-positive patients and anaplastic lymphoma kinase (ALK) TKIs for $A L K$ rearrangement-positive patients. Patients who were treated with nivolumab or pembrolizumab in the second-line setting and subsequently with S-1, with pemetrexed, or with docetaxel with or without ramucirumab as the thirdline treatment between December 1, 2015 and July 31, 2017 were included in the CAP cohort (see online supplementary figure S1). The clinical outcomes for the CAP cohort were compared with those for a control cohort of patients treated with second-line cytotoxic chemotherapy including either S-1, pemetrexed or docetaxel with or without ramucirumab-without preceding ICI therapybetween April 1, 2014 and July 31, 2017. The patients in the control cohort were included from April 1, 2014 in order to collect data on such chemotherapy because nivolumab and pembrolizumab became practically available in Japan from December 2015 and December 2016, respectively, and were then widely used as a second-line treatment. Patient eligibility was confirmed by the WJOG data center.

\section{Outcomes}

The primary end point of the study was ORR. The secondary end points were progression-free survival (PFS) from the first day of treatment with S-1, pemetrexed or docetaxel with or without ramucirumab until disease progression or death due to any cause, OS from the first day of such treatment until death due to any cause, and safety. ORR was assessed by the investigators according to Response Evaluation Criteria in Solid Tumors (V.1.1) and was calculated only for patients with measurable lesions. Safety evaluations included assessment of treatmentrelated select adverse events (AEs), which were defined as AEs with a potential immunologic basis. ${ }^{18}$ AEs were graded according to the National Cancer Institute Common Terminology Criteria for Adverse Events (V.4.0).

\section{Statistical analysis}

Comparisons between the two cohorts were performed with Fisher's exact test for categorical variables and with the Wilcoxon test for continuous variables. Given that we assumed that imbalances in patient characteristics between the two cohorts might exist, we applied propensity score analysis with the inverse probability weighting (IPW) method to minimize the bias due to measured confounders. ${ }^{19}$ The propensity score for each patient was calculated as a probability from a logistic regression model that included all covariates deemed likely to have affected treatment decisions and response-including age, sex, smoking status, Eastern Cooperative Oncology Group performance status, histology, EGFR or ALK genetic alterations, brain metastasis, history of curative radiotherapy and type of chemotherapy (docetaxel with or without ramucirumab, S-1 or pemetrexed). Stabilized weights were calculated for each patient on the basis of the estimated propensity score. ${ }^{19}$ When weighted regression analyses were performed, a robust sandwich variance was used to account for the weighted nature of the sample. $^{19}$

Survival curves for the two cohorts were created with IPW-adjusted Kaplan-Meier plots, and IPW-adjusted HR was calculated with IPW-weighted Cox's proportional hazard models.

We conducted subgroup analysis based on each chemotherapy regimen for the two cohorts. For subgroup analysis of efficacy, the propensity score was calculated by excluding the covariates of histology and history of curative radiotherapy for patients with EGFR or $A L K$ genetic alterations, and by excluding performance status and 
Fig. 1

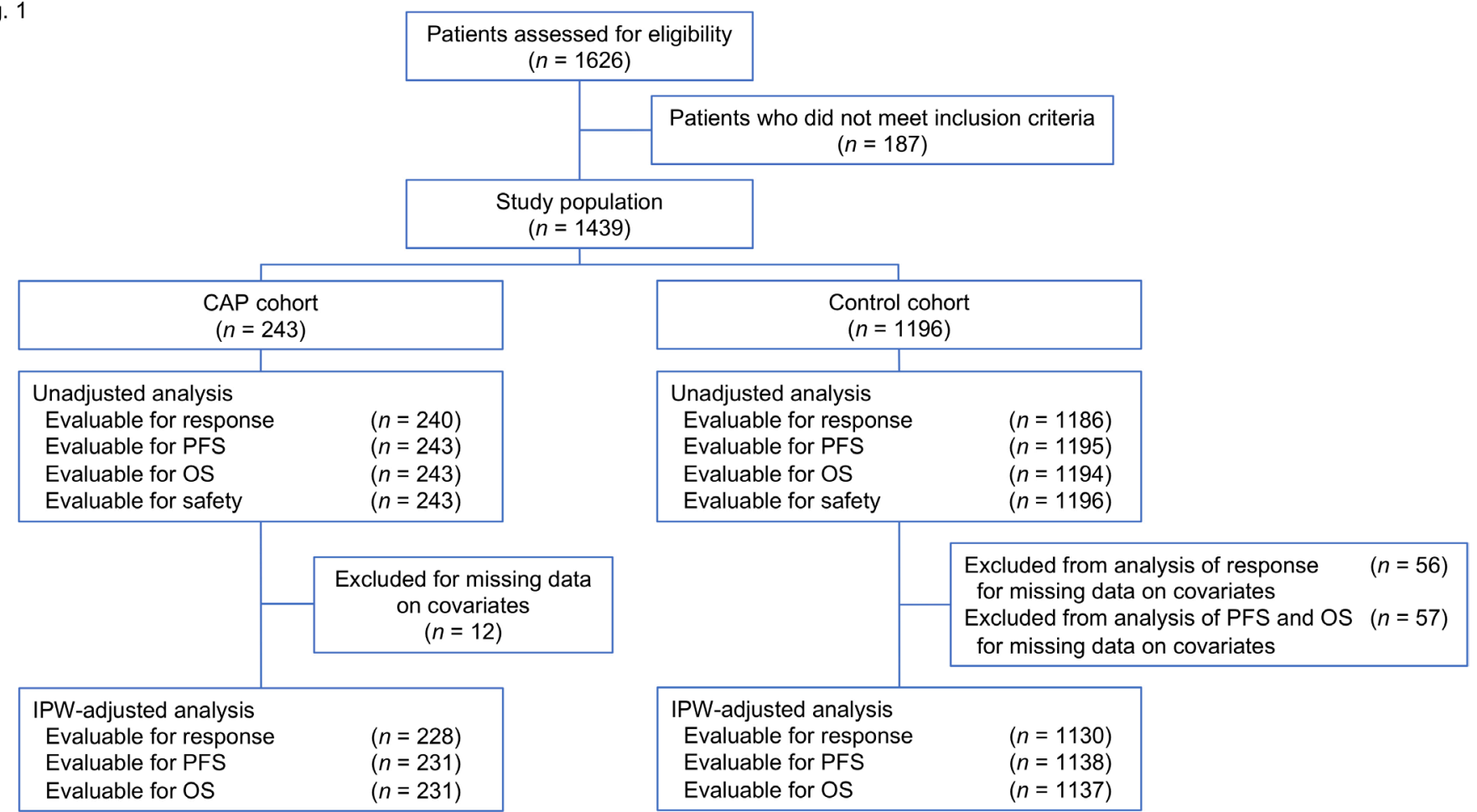

Figure 1 CONSORT diagram. CAP, chemotherapy after PD-1 inhibitor treatment; IPW, inverse probability weighting; OS, overall survival; PFS, progression-free survival.

EGFR or $A L K$ genetic alterations for those with history of curative radiotherapy. We adopted this approach because the logistic regression model used to yield propensity scores was unstable when these covariates were included in the explanatory variables.

All $\mathrm{p}$ values $<0.05$ were considered statistically significant. Clinical data were managed by the WJOG data center. Statistical analysis was performed by an outside contract research organization (EPS, Tokyo, Japan) with SAS (V.9.4) software.

\section{RESULTS}

\section{Patient characteristics}

A total of 1626 patients at 47 institutions was assessed for study eligibility (figure 1). Of these patients, we excluded $187(12 \%)$ individuals who did not meet the inclusion criteria. The remaining 1439 patients were included in the unadjusted analysis. The CAP cohort consisted of 243 patients, with 105 (43\%) having received docetaxel, 77 (32\%) docetaxel plus ramucirumab, 49 (20\%) S-1 and 12 $(5 \%)$ pemetrexed, whereas the control cohort consisted of 1196 patients, with 778 (65\%) having received docetaxel, $94(8 \%)$ docetaxel plus ramucirumab, $174(15 \%) \mathrm{S}-1$ and $150(13 \%)$ pemetrexed. The median follow-up time was 8.1 months (95\% CI 7.5 to 9.4 months) in the CAP cohort and 9.3 months (95\% CI 8.7 to 9.9 months) in the control cohort. Unadjusted patient characteristics and comparisons between the two cohorts are shown in table 1 and online supplementary table S1. The two cohorts differed significantly with respect to age, histology, EGFR or $A L K$ genetic alterations, PD-L1 tumor proportion score (TPS), brain metastasis and type of chemotherapy. To correct for potential imbalances, we performed propensity score analysis. The distribution of propensity score in each cohort is shown in online supplementary figure S2. We evaluated covariate balance with the use of standardized difference. ${ }^{20}$ A standardized difference $<0.1$ would indicate good balance. After IPW adjustment, the covariates were well balanced between the CAP and control cohorts (see online supplementary table S2). Treatment data for first-line chemotherapy, PD-1 inhibitor treatment after first-line chemotherapy and poststudy systemic therapy are provided in online supplementary tables S3 and S4.

\section{Efficacy}

With the IPW method, the ORR for the CAP and control cohorts was found to be $18.9 \%$ and $11.0 \%$, respectively, with the ORR for the CAP cohort being significantly higher than that for the control cohort (ORR ratio 1.71 with $95 \%$ CI 1.19 to 2.46, $\mathrm{p}=0.004$ ) (table 2). IPW-adjusted subgroup analysis according to chemotherapy regimen revealed the ORR for the CAP and control cohorts to be $17.6 \%$ and $11.4 \%$, respectively, for patients treated with docetaxel, and $20.9 \%$ and $18.3 \%$, respectively, for those treated with docetaxel plus ramucirumab. Patients treated with docetaxel or docetaxel plus ramucirumab in the CAP cohort thus had a numerically higher ORR compared with those in the control cohort.

With regard to the results of IPW-adjusted survival analysis, the median PFS for the CAP and control cohorts was 2.8 and 2.7 months, respectively. PFS thus did not 
Table 1 Unadjusted patient characteristics for the chemotherapy after PD-1 inhibitor treatment (CAP) and control cohorts

\begin{tabular}{|c|c|c|c|}
\hline Characteristic & $\begin{array}{l}\text { CAP cohort } \\
(n=243)\end{array}$ & $\begin{array}{l}\text { Control cohort } \\
(n=1196)\end{array}$ & P value* \\
\hline \multicolumn{4}{|l|}{ Age, years $\ddagger$} \\
\hline Median (range) & $68(38-86)$ & $69(30-87)$ & 0.01 \\
\hline$<70$ & $150(61.7)$ & $655(54.8)$ & \multirow[t]{2}{*}{0.06} \\
\hline$\geq 70$ & $93(38.3)$ & $539(45.1)$ & \\
\hline \multicolumn{4}{|l|}{ Sex } \\
\hline Male & $169(69.5)$ & $867(72.5)$ & \multirow[t]{2}{*}{0.35} \\
\hline Female & $74(30.5)$ & $329(27.5)$ & \\
\hline \multicolumn{4}{|l|}{ Smoking status§ } \\
\hline Never & $60(24.7)$ & 278 (23.2) & \multirow[t]{4}{*}{0.78} \\
\hline Past & $102(42.0)$ & $487(40.7)$ & \\
\hline Current & $81(33.3)$ & $423(35.4)$ & \\
\hline Unknown & $0(0.0)$ & $8(0.7)$ & \\
\hline \multicolumn{4}{|l|}{ Histology } \\
\hline Adenocarcinoma & $161(66.3)$ & $859(71.8)$ & \multirow[t]{3}{*}{0.02} \\
\hline Squamous cell carcinoma & $73(30.0)$ & $263(22.0)$ & \\
\hline Other & $9(3.7)$ & $74(6.2)$ & \\
\hline \multicolumn{4}{|l|}{ EGFR mutation status } \\
\hline Positive & $28(11.5)$ & 201 (16.8) & \multirow[t]{3}{*}{$0.04 \rrbracket$} \\
\hline Negative & $177(72.8)$ & $839(70.2)$ & \\
\hline Unknown & $38(15.6)$ & $156(13.0)$ & \\
\hline \multicolumn{4}{|l|}{$A L K$ rearrangement status } \\
\hline Positive & $8(3.3)$ & $13(1.1)$ & \multirow[t]{3}{*}{$0.02 ף$} \\
\hline Negative & $177(72.8)$ & $837(70.0)$ & \\
\hline Unknown & 58 (23.9) & $346(28.9)$ & \\
\hline \multicolumn{4}{|l|}{ PD-L1 TPS ${ }^{* \star}$} \\
\hline$\geq 50 \%$ & $23(9.5)$ & $33(2.8)$ & \multirow[t]{4}{*}{0.008} \\
\hline $1 \%-49 \%$ & $41(16.9)$ & $76(6.4)$ & \\
\hline$<1 \%$ & $32(13.2)$ & $115(9.6)$ & \\
\hline Unknown & $147(60.5)$ & $972(81.3)$ & \\
\hline \multicolumn{4}{|l|}{ ECOG PS††† } \\
\hline $0-1$ & $197(81.1)$ & $1038(86.8)$ & \multirow[t]{3}{*}{$0.054 \ddagger \ddagger$} \\
\hline$\geq 2$ & $34(14.0)$ & $111(9.3)$ & \\
\hline Unknown & $12(4.9)$ & $46(3.8)$ & \\
\hline \multicolumn{4}{|l|}{ Stage§§ } \\
\hline III & $31(12.8)$ & $156(13.0)$ & \multirow[t]{4}{*}{0.94} \\
\hline IV & $170(70.0)$ & $812(67.9)$ & \\
\hline Recurrence after surgery & $33(13.6)$ & $175(14.6)$ & \\
\hline Recurrence after CRT & $9(3.7)$ & $53(4.4)$ & \\
\hline \multicolumn{4}{|l|}{ Brain metastasis $\dagger$} \\
\hline Yes & $65(26.7)$ & $247(20.7)$ & \multirow[t]{2}{*}{0.04} \\
\hline No & $178(73.3)$ & 949 (79.3) & \\
\hline
\end{tabular}


Table 1 Continued

\begin{tabular}{lcc}
\hline Characteristic & $\begin{array}{l}\text { CAP cohort } \\
(\mathbf{n = 2 4 3 )}\end{array}$ & $\begin{array}{l}\text { Control cohort } \\
(\mathbf{n}=\mathbf{1 1 9 6 )}\end{array}$ \\
\hline Docetaxel & $105(43.2)$ & $778(65.1)$ \\
Docetaxel+ramucirumab & $77(31.7)$ & $94(7.9)$ \\
S-1 & $49(20.2)$ & $174(14.5)$ \\
Pemetrexed & $12(4.9)$ & $150(12.5)$ \\
\hline
\end{tabular}

Data are presented as $\mathrm{n}(\%)$ with the exception of median age.

${ }^{*} P$ values were determined with the Wilcoxon test or Fisher's exact test as appropriate.

†At the time of initiation of docetaxel with or without ramucirumab, S-1 or pemetrexed.

$\ddagger$ Two patients in the control cohort had missing data.

$\S N e v e r$ smokers were defined as individuals who had smoked $<100$ cigarettes; past smokers as those who had smoked $\geq 100$ cigarettes but had quit $>1$ year prior to diagnosis; and current smokers as those who had smoked $\geq 100$ cigarettes including at least one within the year prior to diagnosis.

IP values are for comparison between patients positive for EGFR or ALK alterations and those negative or of unknown status.

${ }^{*}$ Data for PD-L1 expression in tumor cells were obtained by immunohistochemistry according to the standard practice of each center. ††One patient in the control cohort had missing data.

$\ddagger \mathrm{P}$ value is for comparison between PS of $0-1$ and PS of 2-4 or unknown.

$\S \S A l l$ patients were classified on the basis of clinical stage according to the seventh edition of the TNM classification.

CRT, chemoradiotherapy; ECOG PS, Eastern Cooperative Oncology Group performance status; TPS, tumor proportion score.

differ significantly between the CAP and control cohorts (IPW-adjusted HR 0.95 with $95 \%$ CI 0.80 to $1.12, \mathrm{p}=0.55$ ) (figure 2). IPW-adjusted Kaplan-Meier curves showed that the median OS for the CAP and control cohorts was 9.2 and 10.4 months, respectively. There was thus no difference in OS between the CAP and control cohorts (IPW-adjusted HR 1.05 with $95 \%$ CI 0.86 to $1.28, \mathrm{p}=0.63$ ) (figure 3).

In the unadjusted analysis, ORR was $17.9 \%$ for the CAP cohort and $10.8 \%$ for the control cohort (ORR ratio 1.66 with $95 \%$ CI 1.21 to $2.28, \mathrm{p}=0.003$ ) (see online supplementary table S5). Unadjusted Kaplan-Meier curves showed that median PFS was 3.2 and 2.7 months (unadjusted HR 0.93 with $95 \%$ CI 0.81 to $1.08, \mathrm{p}=0.36$ ) (see online supplementary figure S3) and that median OS was 9.5 and 10.4 months (unadjusted HR 1.08 with $95 \%$ CI of 0.91 to $-1.28, \mathrm{p}=0.40$ ) (see online supplementary figure S4) for the CAP and control cohorts, respectively.

We performed sensitivity analysis with the use of alternative approaches to evaluate robustness with our findings regarding estimated treatment effect. Multivariable analyses were conducted with a log-linear regression model for response and Cox's proportional hazard model for survival, and with the same covariates as used in the IPW method being included as the explanatory variables. These approaches yielded similar results with those for IPW adjustment (see online supplementary table S6).

\section{Further efficacy analysis in the CAP cohort}

We further evaluated efficacy in the CAP cohort with regard to several factors that potentially could have influenced the response to chemotherapy after ICI treatment (table 3). Data on PD-L1 expression in tumor cells were available for $39.5 \%$ (96 of 243) of patients in the CAP cohort. Of these 96 patients, 23 (24.0\%) individuals had a TPS for PD-L1 $\geq 50 \%, 41(42.7 \%)$ had a TPS of $1 \%-49 \%$ and $32(33.3 \%)$ had a TPS $<1 \%$. The ORR and PFS did not differ between patients with a PD-L1 TPS $\geq 50 \%$ and those with a TPS $<1 \%$. Patients with a PD-L1 TPS of $1 \%-49 \%$ had a lower ORR than did those with a

Table 2 Objective response by inverse probability weighting-adjusted analysis for all patients and according to chemotherapy regimen

\begin{tabular}{|c|c|c|c|c|c|c|c|c|c|c|}
\hline & \multicolumn{2}{|l|}{ All } & \multicolumn{2}{|c|}{ Docetaxel } & \multicolumn{2}{|c|}{ Docetaxel+Ramucirumab } & \multicolumn{2}{|l|}{ S-1 } & \multicolumn{2}{|c|}{ Pemetrexed } \\
\hline & CAP & Control & CAP & Control & CAP & Control & CAP & Control & CAP & Control \\
\hline $\begin{array}{l}\text { No. of } \\
\text { patients }\end{array}$ & 228 & 1130 & 101 & 734 & 61 & 84 & 46 & 151 & 12 & 122 \\
\hline ORR (\%) & 18.9 & 11.0 & 17.6 & 11.4 & 20.9 & 18.3 & 6.0 & 6.0 & 53.6 & 9.8 \\
\hline $\begin{array}{l}\text { ORR ratio } \\
(95 \% \mathrm{Cl}), \mathrm{p} \\
\text { value* }^{*}\end{array}$ & \multicolumn{2}{|c|}{$\begin{array}{l}1.71 \text { (1.19 to } 2.46) \text {, } \\
0.004\end{array}$} & \multicolumn{2}{|c|}{$\begin{array}{l}1.55(0.95 \text { to } 2.51) \text {, } \\
0.08\end{array}$} & \multicolumn{2}{|c|}{1.14 (0.58 to 2.26), 0.70} & \multicolumn{2}{|c|}{$\begin{array}{l}1.01(0.26 \text { to } 3.90) \text {, } \\
0.987\end{array}$} & \multicolumn{2}{|c|}{$\begin{array}{l}5.47(2.32 \text { to } \\
12.91),<0.001\end{array}$} \\
\hline
\end{tabular}

${ }^{*}$ The relative risk $(95 \% \mathrm{Cl})$ is shown for the ORR ratio. The control cohort was the reference cohort for calculation of relative risk, with a relative risk $>1$ thus favoring the CAP cohort.

CAP, chemotherapy after PD-1 inhibitor treatment; ORR, objective response rate. 
Fig. 2

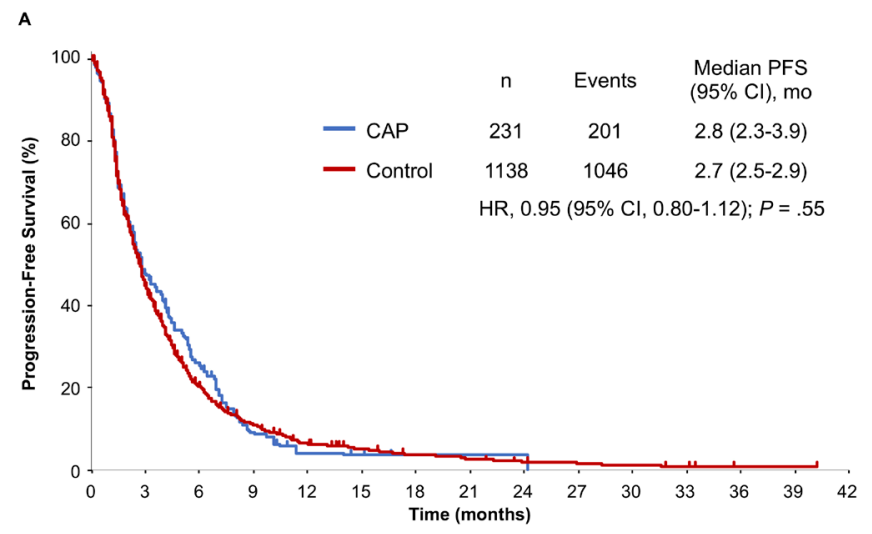

c
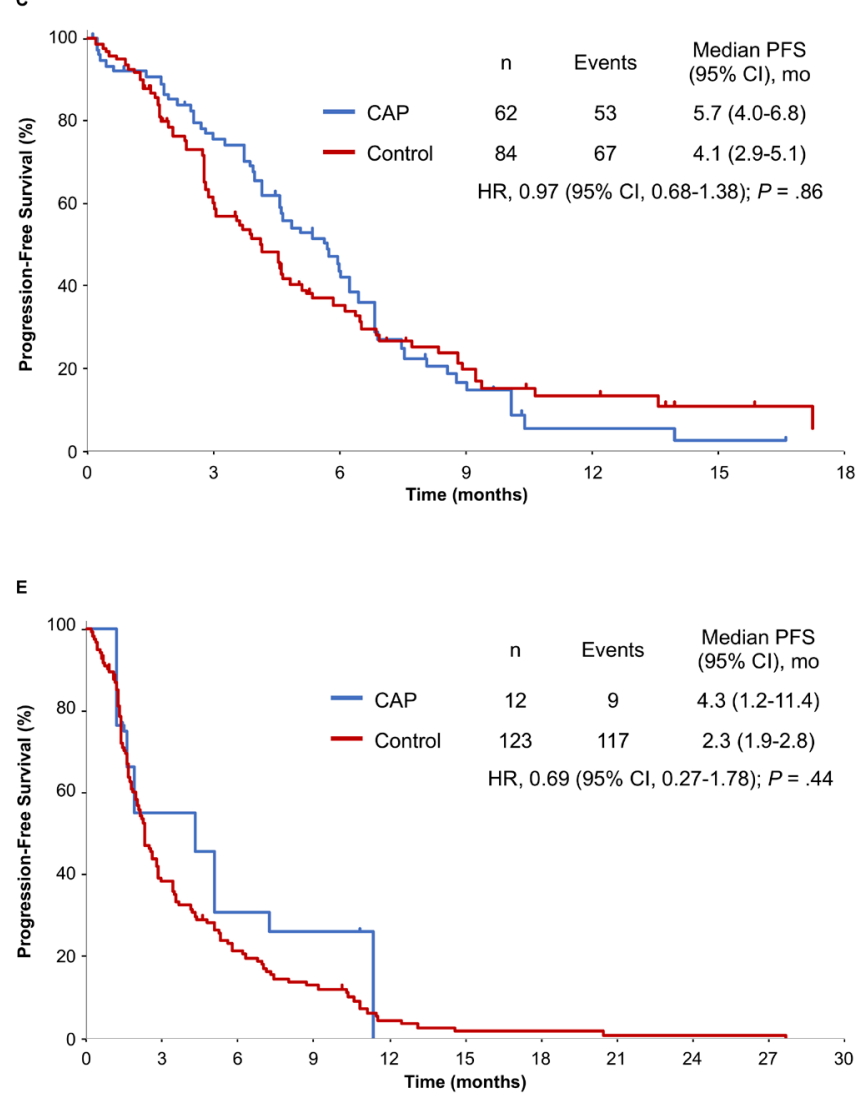

B

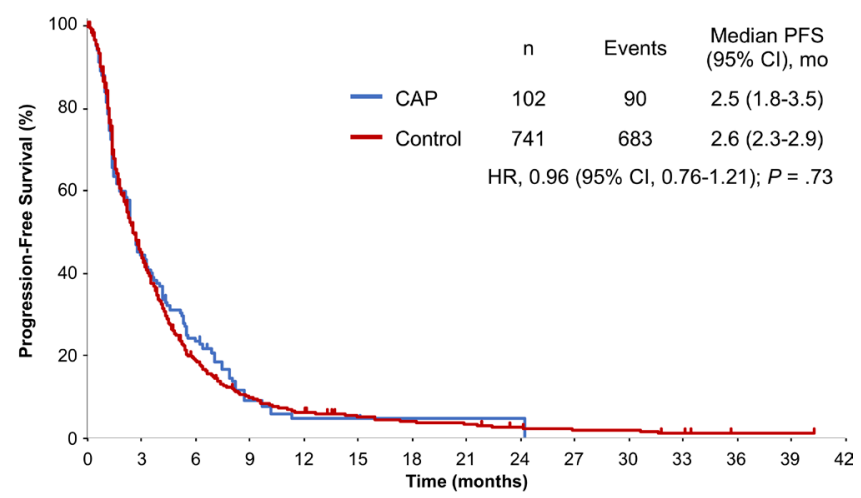

D

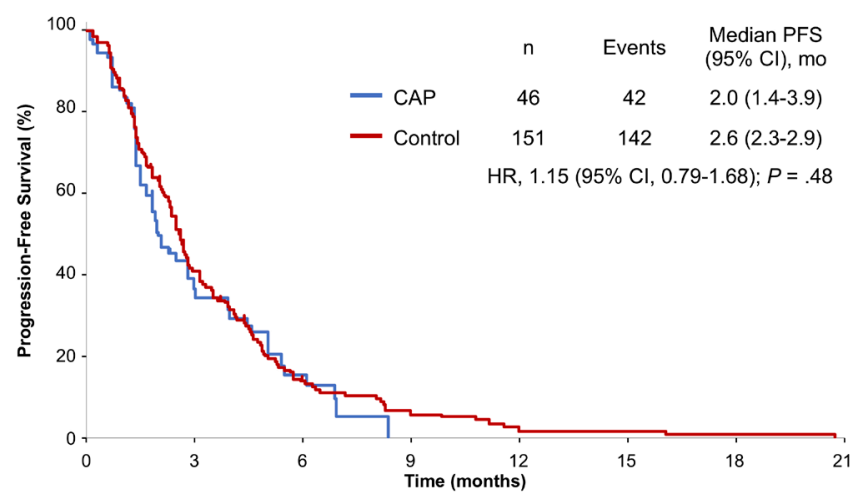

Figure 2 Inverse probability weighting (IPW)-adjusted Kaplan-Meier analysis of progression-free survival (PFS) for the chemotherapy after PD-1 inhibitor treatment (CAP) cohort versus the control cohort. Comparisons are shown for all patients (A) as well as for those treated with docetaxel $(B)$, with docetaxel plus ramucirumab (C), with S-1 (D) or with pemetrexed (E). Vertical lines on the curves denote censoring. mo, month(s).

TPS $<1 \%$, whereas PFS did not differ between these two subgroups. There was no significant difference in ORR or PFS between subgroups of the CAP cohort classified according to duration of PD-1 inhibitor treatment, type of response to PD-1 inhibitor treatment, the interval between the last dose of PD-1 inhibitor and the start of subsequent chemotherapy, EGFR or $A L K$ alteration status or history of curative radiotherapy.

\section{Safety}

Finally, we examined whether the CAP cohort experienced increased toxicity, given that small series of patients with melanoma or NSCLC were previously found to experience severe systemic toxicities during treatment with TKIs subsequent to that with ICIs. ${ }^{21-23}$ Treatmentrelated select AEs for chemotherapy, PD-1 inhibitors and each chemotherapy regimen separately are listed in table 4 and online supplementary tables S7-11. AEs of any grade for chemotherapy that showed a significantly higher incidence in the CAP cohort than in the control cohort included stomatitis $(14.4 \%$ vs $8.8 \%, \mathrm{p}=0.009)$ and hypothyroidism ( $1.2 \%$ vs $0 \%, \mathrm{p}=0.005)$. Subgroup analysis according to chemotherapy regimen revealed 
Fig. 3
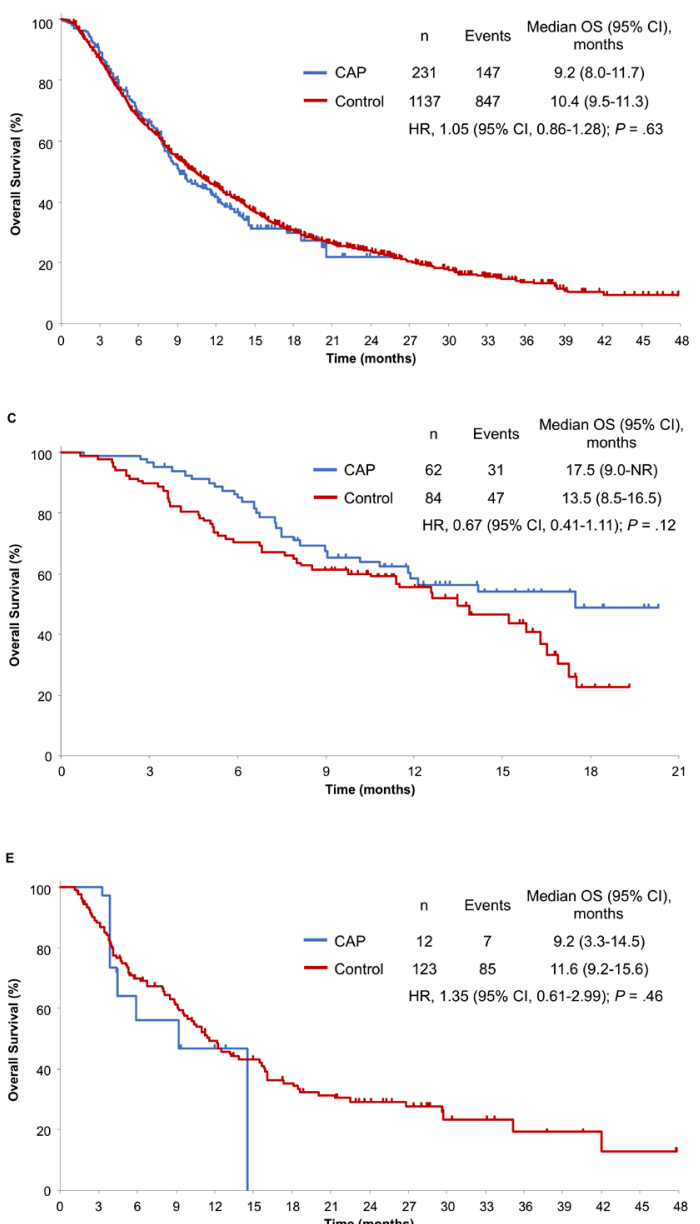

в
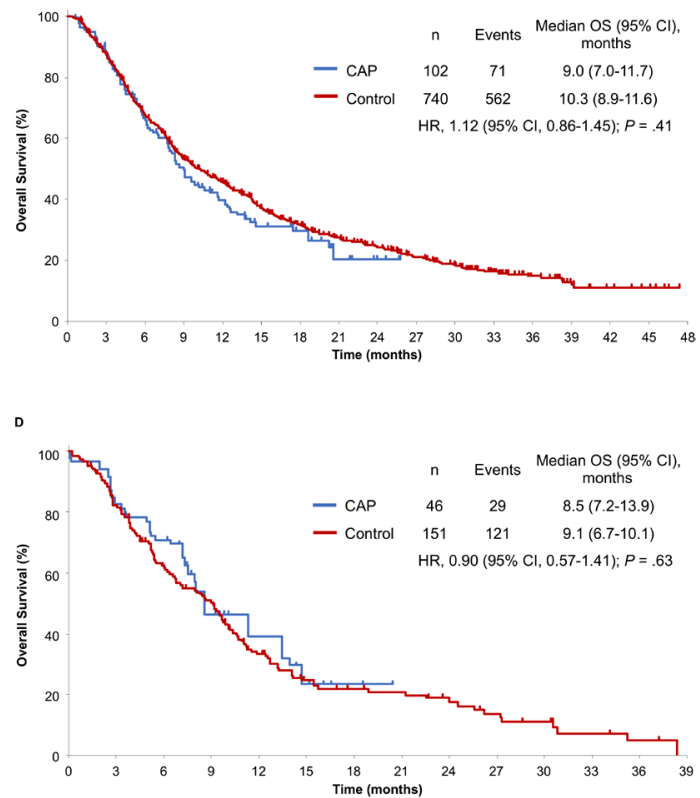

Figure 3 Inverse probability weighting (IPW)-adjusted Kaplan-Meier analysis of overall survival (OS) for the chemotherapy after PD-1 inhibitor treatment (CAP) cohort versus the control cohort. Comparisons are shown for all patients (A) as well as for those treated with docetaxel $(B)$, with docetaxel plus ramucirumab $(C)$, with S-1 (D) or with pemetrexed (E). Vertical lines on the curves denote censoring. NR, not reached.

no significant differences in the incidence of any-grade stomatitis or hypothyroidism between the CAP and control cohorts, whereas the incidence of an increase in total bilirubin level of any grade in patients treated with docetaxel or of hyperglycemia and increases in aspartate aminotransferase (AST) and alanine aminotransferase (ALT) levels of any grade in those treated with S-1 was significantly higher in the CAP cohort than in the control cohort. All treatment-related deaths were due to pneumonitis: one patient $(0.4 \%)$ in the CAP cohort and seven patients $(0.6 \%)$ in the control cohort.

\section{DISCUSSION}

In this multicenter retrospective cohort study for advanced NSCLC based on propensity score analysis with the IPW method, we found that CAP was associated with a higher ORR, but no PFS or OS benefit, compared with chemotherapy alone.

The choice of treatment in real-world clinical practice can be influenced by patient characteristics. ${ }^{16}$ Indeed, in our study, many important baseline characteristics differed significantly between the CAP and control cohorts. The presence of such an imbalance can lead to a biased estimate of treatment effect. ${ }^{15}$ We therefore applied the propensity score to balance the distribution of these measured covariates between the cohorts. ${ }^{24}$ To our knowledge, our study is the only one to date to describe clinical outcomes of patients treated sequentially with PD-1 inhibitors and chemotherapy with the use of the propensity score to address this bias.

Several different propensity score-based methods have been developed. ${ }^{15-17} 19$ The most common method is propensity score matching, in which patients with similar propensity scores in the treatment and control groups are matched. ${ }^{15} 17$ One disadvantage of such matching, however, is that unmatched patients are excluded from the analysis, leading to a reduced generalizability and accuracy of the results. ${ }^{15}$ This disadvantage is overcome with the IPW method, which generates a weight based on the propensity score. This method can include all patients in the analysis and generates a pseudopopulation in which the measured confounding variables are balanced between the groups. ${ }^{16}$ We thus applied the IPW method in the present study. 


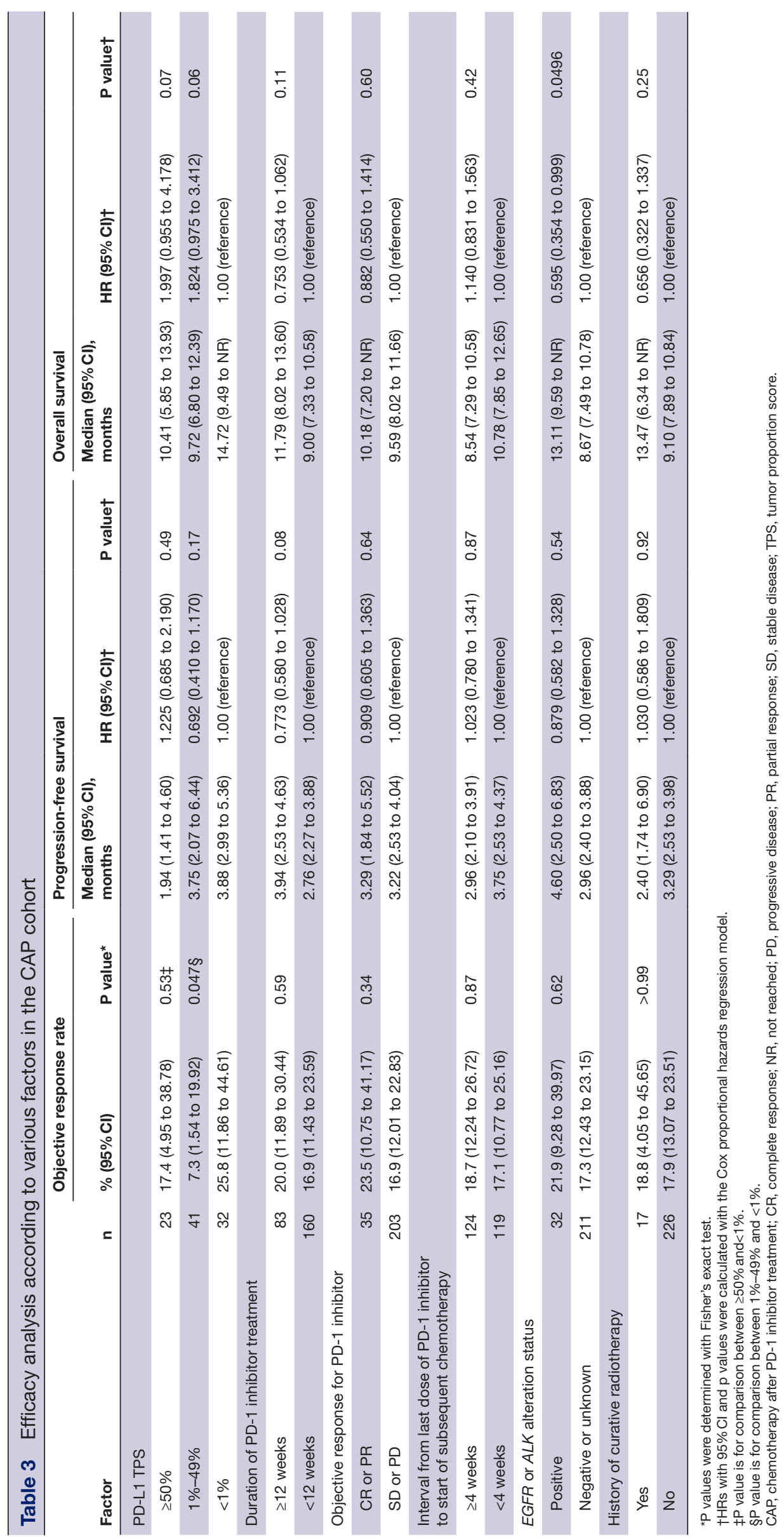


Table 4 Treatment-related select adverse events for chemotherapy

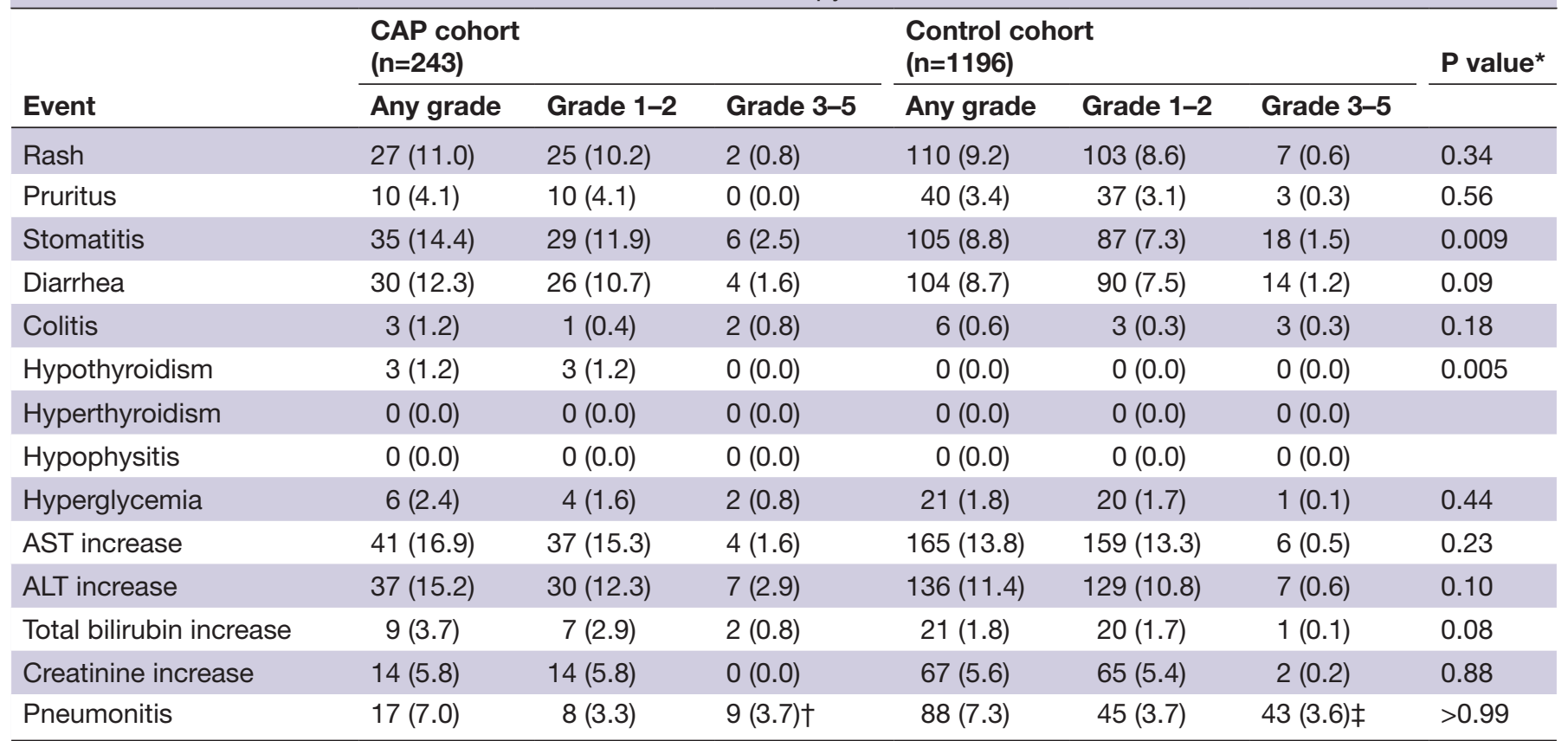

Data are presented as $\mathrm{n}(\%)$.

${ }^{*} P$ values were determined by Fisher's exact test and are for between-cohort comparisons of rates of any-grade adverse events.

†Data include one patient with treatment-related pneumonitis of grade 5.

¥Data include seven patients with treatment-related pneumonitis of grade 5 .

CAP, chemotherapy after PD-1 inhibitor treatment; AST, aspartate aminotransferase; ALT, alanine aminotransferase.

The primary end point of ORR was met in our study, providing support for previous suggestions that chemotherapy after ICI exposure confers an improved response in patients with NSCLC. ${ }^{10}{ }^{11}$ However, this result did not translate into a PFS or OS advantage, indicating that CAP does not confer a durable antitumor response. These findings are consistent with those of a previous study in which chemotherapy after ICIs did not show a PFS benefit despite an increased ORR. ${ }^{10}$ Although the difference in treatment line between the CAP (third line) and control (second line) cohorts might have influenced the PFS and OS results, one possible explanation for this lack of a sustained survival benefit is suggested by pharmacokinetics data for patients with advanced NSCLC showing that binding of nivolumab to $\mathrm{T}$ cells remained apparent for $>2$ months after the last infusion regardless of subsequent treatment and that the percentage binding decreased in a time-dependent manner. ${ }^{2526}$ Such prolonged binding of PD-1 inhibitors after their discontinuation may thus give rise to a transient synergism in antitumor effect with subsequent chemotherapy, with this synergism decreasing as the percentage binding of the inhibitors to $\mathrm{T}$ cells declines.

An important question that follows from the results of recent randomized studies is whether combinations of ICIs and chemotherapy have greater efficacy when administered concurrently than when given sequentially ICIs followed by chemotherapy in previously untreated patients with metastatic NSCLC, especially in those with a PD-L1 TPS $\geq 50 \% .{ }^{12} 132728$ The concurrent administration of an ICI and cytotoxic chemotherapy is now widely adopted as a first-line treatment for advanced NSCLC. ${ }^{1328}$ Moreover, phase III studies have demonstrated a survival benefit for pembrolizumab monotherapy relative to platinum-based chemotherapy and support the use of pembrolizumab monotherapy as a first-line treatment for advanced NSCLC in patients with a PD-L1 TPS $\geq 1 \% .{ }^{12} 14$ Approximately $40 \%$ of patients received chemotherapy after disease progression during pembrolizumab monotherapy in one of these studies. ${ }^{14}$ Given that more and more patients receive an ICI alone or in combination with chemotherapy as a first-line treatment, the efficacy and safety of chemotherapy after the preceding administration of ICI treatment are key factors, and our findings now provide important information for such patients. In the present study, the CAP cohort did not show a durable clinical benefit-and, in particular, the treatment did not show a higher efficacy in patients with a PD-L1 TPS $\geq 50 \%$ than in those with a TPS $<1 \%$. On the basis of these findings and the pharmacokinetics data described above, concurrent administration of ICIs and chemotherapy might be a more promising therapeutic approach than the sequential strategy for maximizing the synergistic clinical activities of ICIs and chemotherapy. A phase III study to investigate whether the concurrent or sequential strategy in the first-line setting is more efficacious for NSCLC is currently ongoing (ClinicalTrials.gov identifier NCT03793179).

We observed a significantly higher incidence of stomatitis in the CAP cohort than in the control cohort, likely 
reflecting the higher proportion of patients who received docetaxel plus ramucirumab in the former than in the latter cohort. Previous studies have shown that stomatitis occurs at a higher frequency in patients treated with docetaxel plus ramucirumab than in those receiving docetaxel alone. ${ }^{8}{ }^{29}$ Subgroup analysis according to chemotherapy regimen did not reveal any significant differences in the occurrence of stomatitis between the two cohorts. On the other hand, we detected significantly higher rates of hepatotoxicity, including elevation of total bilirubin, AST and ALT levels, in the CAP cohort than in the control cohort among patients treated with docetaxel or S-1. Consistent with previous findings, these results suggest that careful monitoring for hepatotoxicity may be warranted in patients treated with chemotherapy after PD-1 inhibitors. ${ }^{22}$

Our study has several limitations. First, our findings are based on a retrospective cohort analysis performed with electronic medical records, with their inherent variability in accuracy and data availability. In particular, the interval for imaging was highly variable, representing a bias for PFS assessment. However, comparison of the efficacy of chemotherapy with or without prior ICI treatment is possible only with such a retrospective design. Second, we did not account for PD-L1 expression as a confounder. PD-L1 testing currently provides important information for selection of patients most likely to benefit from ICI therapy, although there was previously little evidence that PD-L1 status was associated with ICI efficacy in NSCLC. Nivolumab was widely administered as a second-line treatment regardless of PD-L1 expression level until 2017, given that no diagnostic kits had been commercially available in Japan. PD-L1 TPS thus did not critically affect treatment decisions for most patients in the CAP cohort treated between December 1, 2015 and July 31, 2017, and we therefore did not include it as a covariate in our study. Third, the fact that the present study inherently compares second-line chemotherapy (control cohort) with thirdline chemotherapy (CAP cohort) might have influenced the PFS and OS results.

\section{CONCLUSIONS}

After adjustment for selection bias by propensity score analysis according to the IPW method, our study has shown that CAP was associated with a higher ORR compared with chemotherapy alone, with the primary end point of ORR thus being achieved. However, PFS and OS did not differ between the two cohorts. Our findings indicate that the preceding administration of PD-1 inhibitors may give rise to a synergistic antitumor effect with chemotherapy, but that this effect is likely not persistent. CAP therefore does not appear to give rise to durable tumor control and a consequent survival benefit.

\section{Author affiliations}

${ }^{1}$ Department of Medical Oncology, Kindai University Faculty of Medicine, OsakaSayama, Japan
${ }^{2}$ Clinical Research Center, Kindai University Hospital, Osaka-Sayama, Japan ${ }^{3}$ Division of Thoracic Oncology, Shizuoka Cancer Center, Nagaizumi, Japan ${ }^{4}$ Department of Thoracic Oncology, Aichi Cancer Center Hospital, Nagoya, Japan ${ }^{5}$ Department of Medical Oncology, Kishiwada City Hospital, Kishiwada, Japan ${ }^{6}$ Department of Respiratory Medicine, Kobe City Medical Center General Hospital, Kobe, Japan

${ }^{7}$ Department of Thoracic Oncology, National Hospital Organization Kyushu Cancer Center, Fukuoka, Japan

${ }^{8}$ Department of Pulmonary Medicine, Sendai Kousei Hospital, Miyagi, Japan

${ }^{9}$ Department of Thoracic Oncology, National Hospital Organization Shikoku Cancer Center, Matsuyama, Japan

${ }^{10}$ Research Institute for Diseases of The Chest, Graduate School of Medical Sciences, Kyushu University, Fukuoka, Japan

${ }^{11}$ Third Department of Internal Medicine, Wakayama Medical University, Wakayama, Japan

${ }^{12}$ Division of Respirology, NTT Medical Center Tokyo, Tokyo, Japan

${ }^{13}$ Department of Thoracic Oncology, Osaka International Cancer Institute, Osaka, Japan

${ }^{14}$ Respiratory Center, Matsusaka Municipal Hospital, Matsusaka, Japan

${ }^{15}$ Department of Respiratory Disease, National Hospital Organization Fukuoka Higashi Medical Center, Koga, Japan

${ }^{16}$ Department of Respiratory Medicine, lizuka Hospital, lizuka, Japan

${ }^{17}$ Department of Respiratory Medicine, Kumamoto University Hospital, Kumamoto, Japan

${ }^{18}$ Department of Thoracic Oncology, National Hospital Organization Osaka Toneyama Medical Center, Toyonaka, Japan

${ }^{19}$ Department of Respiratory Medicine, Okazaki City Hospital, Okazaki, Japan

AcknowledgmentsWe thank the staff at all investigational sites, as well as the data managers and other support staff of West Japan Oncology Group (WJOG), especially Koji Takeda and Shinichiro Nakamura.

Funding This study was supported by WJOG, a non-profit organization supported by unrestricted donations from several pharmaceutical companies and personal property.

Competing interests RK has received lecture fees from Bristol-Myers Squibb. HH has received honoraria from AstraZeneca K.K., Boehringer Ingelheim Japan, Bristol-Myers Squibb, Chugai Pharmaceutical, Eli Lilly Japan K.K., MSD K.K., Ono Pharmaceutical, Pfizer Japan, Novartis Pharma K.K., Merck Serono and Taiho Pharmaceutical; and research funding from AbbVie, AC MEDICAL, Astellas Pharma, AstraZeneca K.K., Boehringer Ingelheim Japan, Bristol-Myers Squibb, Daiichi Sankyo, Eisai, Eli Lilly Japan K.K., EPS Associates, GlaxoSmithKline K.K., Japan Clinical Research Operations, Kyowa Hakko Kirin, Merck Serono, MSD K.K., Novartis Pharma K.K., Ono Pharmaceutical, Otsuka Pharmaceutical, PAREXEL International, Pfizer Japan, PPD-SNBL K.K., Quintiles Transnational Japan K.K., Taiho Pharmaceutical, Takeda Pharmaceutical and Yakult Honsha. YC has received honoraria from Chugai Pharmaceutical. DF has received lecture fees from Eli Lilly Japan K.K., Boehringer Ingelheim Japan, Ono Pharmaceutical, BristolMyers Squibb, Chugai Pharmaceutical, Taiho Pharmaceutical, Novartis Pharma K.K., MSD K.K. and AstraZeneca K.K.; honoraria from Eli Lilly Japan K.K., Ono Pharmaceutical, Bristol-Myers Squibb, Taiho Pharmaceutical and AstraZeneca K.K.; and research funding from AstraZeneca K.K. RT has received honoraria from AstraZeneca K.K., Bristol-Myers Squibb, Chugai Pharmaceutical, Eli Lilly Japan K.K., Kyowa Hakko Kirin, MSD K.K., Boehringer Ingelheim Japan, Nippon Kayaku, Ono Pharmaceutical and Taiho Pharmaceutical. AN has received honoraria from MSD K.K., AstraZeneca K.K. and Chugai Pharmaceutical. TK has received honoraria from Chugai Pharmaceutical, AstraZeneca K.K., Eli Lilly Japan K.K., Taiho Pharmaceutical, Bristol-Myers Squibb, Ono Pharmaceutical, MSD K.K., Pfizer Japan, Kyowa Hakko Kirin, Boehringer Ingelheim Japan, Nippon Kayaku; and research funding from Chugai Pharmaceutical, AstraZeneca K.K., Eli Lilly Japan K.K., Bristol-Myers Squibb, Pfizer Japan and Merck Serono. KT has received honoraria from Chugai Pharmaceutical, AstraZeneca K.K., Boehringer Ingelheim Japan, Eli Lilly Japan K.K., Ono Pharmaceutical. ST has received honoraria from Chugai Pharmaceutical, Novartis Pharma K.K., AstraZeneca K.K. and Taiho Pharmaceutical; and consulting fees from Boehringer Ingelheim Japan and AbbVie. KU has received lecture fees from AstraZeneca K.K., Boehringer Ingelheim Japan, Bristol-Myers Squibb, MSD K.K., Novartis Pharma K.K., Ono Pharmaceutical, Pfizer Japan and Taiho Pharmaceutical. KN has received honoraria from Chugai Pharmaceutical, AstraZeneca K.K., Boehringer Ingelheim Japan, MSD K.K., Bristol-Myers Squibb, Ono Pharmaceutical, Eli Lilly Japan K.K. and Novartis Pharma K.K.; and research funding from Boehringer Ingelheim Japan. $\mathrm{OH}$ has received honoraria from Eli Lilly 
Japan K.K., Novartis Pharma K.K., AstraZeneca K.K. and Boehringer Ingelheim Japan; and research funding from Novartis Pharma K.K., GlaxoSmithKline K.K., Daiichi Sankyo, Bayer Yakuhin, KYORIN Pharmaceutical, Boehringer Ingelheim Japan and Ono Pharmaceutical. YA has received honoraria from AstraZeneca K.K.; and research funding from Chugai Pharmaceutical. NY has received honoraria from AstraZeneca K.K., Boehringer Ingelheim Japan, Bristol-Myers Squibb, Chugai Pharmaceutical, Eli Lilly Japan K.K., MSD K.K., Novartis Pharma K.K., Ono Pharmaceutical, Pfizer Japan, Takeda Pharmaceutical and Taiho Pharmaceutical; research funding from Boehringer Ingelheim Japan, Chugai Pharmaceutical, Eli Lilly Japan K.K. and MSD K.K.; and consulting or advisory fees from AstraZeneca K.K., Boehringer Ingelheim Japan, Bristol-Myers Squibb, Chugai Pharmaceutical, Eli Lilly Japan K.K., MSD K.K., Novartis Pharma K.K., Ono Pharmaceutical, Pfizer Japan and Takeda Pharmaceutical. KN has received honoraria from Astellas Pharma, AstraZeneca K.K., Bristol-Myers Squibb, Chugai Pharmaceutical, Clinical Trial, Eli Lilly Japan K.K., MSD K.K., Nichi-Iko Pharmaceutical, Boehringer Ingelheim Japan, Novartis Pharma K.K., Ono Pharmaceutical, Pfizer Japan, Reno. Medical K.K. and Sym Bio Pharmaceuticals; research funding from A2 Healthcare, AbbVie, Astellas Pharma, Bristol-Myers Squibb, Chugai Pharmaceutical, Daiichi Sankyo, Eisai, Eli Lilly Japan K.K., EP-CRSU, GRITSTONE ONCOLOGY, ICON Japan K.K., inVentiv Health Japan, MSD K.K., Linical, Boehringer Ingelheim Japan, Novartis Pharma K.K., Ono Pharmaceutical, PAREXEL International, Pfizer Japan, Quintiles Quintiles, Taiho Pharmaceutical and Takeda Pharmaceutical; and consulting or advisory fees from Astellas Pharma, Takeda Pharmaceutical and Ono Pharmaceutical.

\section{Patient consent for publication Not required.}

Ethics approval The study (WJOG10217L) was approved by the protocol review committee of WJOG and the institutional review board of each participating institution, and it was registered in the UMIN database (ID 000029576).

Provenance and peer review Not commissioned; externally peer reviewed.

Data availability statement Data are available on reasonable request. The datasets used and/or analyzed during the current study are available from the corresponding author on reasonable request.

Open access This is an open access article distributed in accordance with the Creative Commons Attribution Non Commercial (CC BY-NC 4.0) license, which permits others to distribute, remix, adapt, build upon this work non-commercially, and license their derivative works on different terms, provided the original work is properly cited, appropriate credit is given, any changes made indicated, and the use is non-commercial. See http://creativecommons.org/licenses/by-nc/4.0/.

\section{ORCID iD}

Hidetoshi Hayashi http://orcid.org/0000-0001-8787-5587

\section{REFERENCES}

1 Brahmer J, Reckamp KL, Baas P, et al. Nivolumab versus docetaxel in advanced squamous-cell Non-Small-Cell lung cancer. $N$ Engl J Med Overseas Ed 2015;373:123-35.

2 Borghaei H, Paz-Ares L, Horn L, et al. Nivolumab versus docetaxel in advanced nonsquamous non-small-cell lung cancer. N Engl J Med 2015;373:1627-39.

3 Herbst RS, Baas P, Kim D-W, et al. Pembrolizumab versus docetaxel for previously treated, PD-L1-positive, advanced non-small-cell lung cancer (KEYNOTE-010): a randomised controlled trial. Lancet 2016;387:1540-50.

4 Rittmeyer A, Barlesi F, Waterkamp D, et al. Atezolizumab versus docetaxel in patients with previously treated non-small-cell lung cancer (OAK): a phase 3, open-label, multicentre randomised controlled trial. Lancet 2017;389:255-65.

5 Shepherd FA, Dancey J, Ramlau R, et al. Prospective randomized trial of docetaxel versus best supportive care in patients with non-small-cell lung cancer previously treated with platinum-based chemotherapy. J Clin Oncol 2000;18:2095-103.

6 Fossella FV, DeVore R, Kerr RN, et al. Randomized phase III trial of docetaxel versus vinorelbine or ifosfamide in patients with advanced non-small-cell lung cancer previously treated with platinumcontaining chemotherapy regimens. The TAX 320 Non-Small Cell Lung Cancer Study Group. J Clin Oncol 2000;18:2354-62.

7 Hanna N, Shepherd FA, Fossella FV, et al. Randomized phase III trial of pemetrexed versus docetaxel in patients with non-small-cell lung cancer previously treated with chemotherapy. J Clin Oncol 2004;22:1589-97.

8 Garon EB, Ciuleanu T-E, Arrieta O, et al. Ramucirumab plus docetaxel versus placebo plus docetaxel for second-line treatment of stage IV non-small-cell lung cancer after disease progression on platinum-based therapy (REVEL): a multicentre, double-blind, randomised phase 3 trial. Lancet 2014;384:665-73.

9 Nokihara H, Lu S, Mok TSK, et al. Randomized controlled trial of S-1 versus docetaxel in patients with non-small-cell lung cancer previously treated with platinum-based chemotherapy (East Asia S-1 trial in lung cancer). Ann Oncol 2017;28:2698-706.

10 Park SE, Lee SH, Ahn JS, et al. Increased response rates to salvage chemotherapy administered after PD-1/PD-L1 inhibitors in patients with Non-Small cell lung cancer. J Thorac Oncol 2018;13:106-11.

11 Schvartsman G, Peng SA, Bis G, et al. Response rates to singleagent chemotherapy after exposure to immune checkpoint inhibitors in advanced non-small cell lung cancer. Lung Cancer 2017;112:90-5.

12 Reck M, Rodríguez-Abreu D, Robinson AG, et al. Pembrolizumab versus chemotherapy for PD-L1-Positive Non-Small-Cell lung cancer. N Engl J Med 2016;375:1823-33.

13 Gandhi L, Rodríguez-Abreu D, Gadgeel S, et al. Pembrolizumab plus chemotherapy in metastatic Non-Small-Cell lung cancer. N Engl J Med 2018;378:2078-92.

14 TSK M, YL W, Kudaba I, et al. Pembrolizumab versus chemotherapy for previously untreated, PD-L1-expressing, locally advanced or metastatic non-small-cell lung cancer (KEYNOTE-042): a randomised, open-label, controlled, phase 3 trial. Lancet 2019;393:1819-30

15 Deb S, Austin PC, Tu JV, et al. A review of Propensity-Score methods and their use in cardiovascular research. Can J Cardiol 2016;32:259-65.

16 Elze MC, Gregson J, Baber U, et al. Comparison of propensity score methods and covariate adjustment: evaluation in 4 cardiovascular studies. J Am Coll Cardiol 2017;69:345-57.

17 Benedetto U, Head SJ, Angelini GD, et al. Statistical primer: propensity score matching and its alternatives $†$. Eur $J$ Cardiothorac Surg 2018;53:1112-7.

18 Weber JS, Hodi FS, Wolchok JD, et al. Safety profile of nivolumab monotherapy: a pooled analysis of patients with advanced melanoma. J Clin Oncol 2017;35:785-92.

19 Hernán MA, Robins JM. Causal inference. Boca Raton: Chapman \& Hall/CRC (forthcoming), 2019.

20 Austin PC. Balance diagnostics for comparing the distribution of baseline covariates between treatment groups in propensity-score matched samples. Stat Med 2009;28:3083-107.

21 Harding JJ, Pulitzer M, Chapman PB. Vemurafenib sensitivity skin reaction after ipilimumab. N Engl J Med 2012;366:866-8.

22 Lin JJ, Chin E, Yeap BY, et al. Increased hepatotoxicity associated with sequential immune checkpoint inhibitor and crizotinib therapy in patients with Non-Small cell lung cancer. $J$ Thorac Oncol 2019;14:135-40.

23 Johnson DB, Wallender EK, Cohen DN, et al. Severe cutaneous and neurologic toxicity in melanoma patients during vemurafenib administration following anti-PD-1 therapy. Cancer Immunol Res 2013;1:373-7.

24 Austin PC. The use of propensity score methods with survival or time-to-event outcomes: reporting measures of effect similar to those used in randomized experiments. Stat Med 2014;33:1242-58.

25 Osa A, Uenami T, Koyama S, et al. Clinical implications of monitoring nivolumab immunokinetics in non-small cell lung cancer patients. JCl Insight 2018;3.

26 Brahmer JR, Drake CG, Wollner I, et al. Phase I study of single-agent anti-programmed death-1 (MDX-1106) in refractory solid tumors: safety, clinical activity, pharmacodynamics, and immunologic correlates. J Clin Oncol 2010;28:3167-75.

27 Socinski MA, Jotte RM, Cappuzzo F, et al. Atezolizumab for firstline treatment of metastatic Nonsquamous NSCLC. N Engl J Med 2018;378:2288-301.

28 Paz-Ares L, Luft A, Vicente D, et al. Pembrolizumab plus chemotherapy for squamous Non-Small-Cell lung cancer. $N$ Engl J Med 2018;379:2040-51.

29 Yoh K, Hosomi Y, Kasahara K, et al. A randomized, double-blind, phase II study of ramucirumab plus docetaxel vs placebo plus docetaxel in Japanese patients with stage IV non-small cell lung cancer after disease progression on platinum-based therapy. Lung Cancer 2016;99:186-93. 